\title{
Antifounded Coinduction in Type Theory
}

\author{
Tarmo Uustalu \\ Institute of Cybernetics at Tallinn University of Technology \\ Akadeemia tee 21. FF-12618 Tallinn, Estonia \\ tarmo@cs.ioc.ee
}

Capretta, Vene and myself have previously [2] studied antifounded algebras as algebras supporting a form of coinduction in a category-theoretic setting, arising as the dual concept of wellfounded coalgebras.

Wellfounded coalgebras are a category theorist's take on coalgebras supporting induction, introduced by Taylor [4]. Informally, wellfoundedness of a coalgebra means that a subobject of its carrier, called the wellfounded part, is isomorphic to the carrier. Under mild conditions, wellfounded coalgebras are the same as recursive coalgebras in the sense of Osius [3], i.e., coalgebras supporting recursion.

Categorical wellfounded coalgebras are intimately related to the type-theoretic method of Bove and Capretta [1] for justifying function definitions by recursion and proofs by induction on a coalgebra. The idea of the method is this: One defines the wellfounded part of the coalgebra in terms of an inductively defined predicate of the carrier, to then rewrite the given "general" recursive definition or inductive proof on the carrier as a "structural" recursive definition or inductive proof on the wellfounded part (these are systematic constructions). Separately, one establishes (in some way) that the carrier is contained in the wellfounded part.

In this paper, I attempt to tell a type-theoretic story of antifounded coinduction. Coinductive types are infamous by posing problems for both intensional and extensional type theory. Hence, it is only to be expected that not everything will work out as smoothly as for wellfounded induction. Nonetheless, a type-theoretic formulation of antifounded coinduction is both possible and meaningful. It is a coinduction-like proof principle for algebras that are antifounded in the sense that a particular quotient of the carrier, called the antifounded quotient, is isomorphic to the carrier. Type-theoretically, this set is specified as a quotient of the carrier by a coinductively defined equivalence relation. I give examples of the use of this proof principle and explain why an algebra supporting coinduction does not necessarily support corecursion.

Acknowledgement This work was supported by the Estonian Science Foundation grant no. 6940 .

\section{References}

[1] A. Bove, V. Capretta. Modelling general recursion in type theory. Math. Struct. in Comput. Sci., 14(4):671-708, 2005.

[2] V. Capretta, T. Uustalu, V. Vene. Corecursive algebras: a study of general structured corecursion. In M. Oliveira, J. Woodcock, eds., Revised Selected Papers from 12th Brazilian Symp. on Formal Methods, SBMF 2009 (Gramado, RS, Aug. 2009), v. 5902 of Lect. Notes in Comput. Sci., pp. 84-100. Springer, 2009

[3] G. Osius. Categorical set theory: a characterization of the category of sets. J. of Pure and Appl. Algebra, 4:79-119, 1974.

[4] P. Taylor. Practical foundations of mathematics, v. 59 of Cambridge Studies in Advanced Mathematics, Cambridge Univ. Press, 1999. 